\title{
A APLICAÇÃO DE ANIMAÇÕES NO ENSINO DE FÍSICA
}

\author{
Juliano Schimiguel - UNICSUL - schimiguel@gmail.com Waltrudes Everton \\ Sanches - UNICSUL - waltrudeseverton@hotmail.com Marcos Roberto \\ Andreasi - Unianchieta - marcosrobertoandreasi@gmail.com Paulo Henrique \\ de Siqueira - UNICSUL - paulohenriqu@hotmail.com
}

\begin{abstract}
Resumo: Este trabalho se propôs a verificar se o uso de animações interativas computacionais, na função de organizadores prévios, pode favorecer os processos de ensino e aprendizagem dos conceitos da energia mecânica. Para tanto, foi desenvolvida uma animação interativa, denominada Energia no Bate - Estaca, e empregado um estudo que avaliou a influência da utilização desse recurso junto a alunos de uma escola pública de Ensino Médio. Os resultados obtidos sugerem que o uso de animações interativas como organizadores prévios é uma alternativa válida e eficaz para favorecer o processo ensino-aprendizagem dos conceitos da Energia Mecânica.
\end{abstract}

Palavras-chave: animação interativa; simulação; energia; ensino da física.

\section{APPLICATION OF ANIMATIONS IN PHYSICS TEACHING}

Abstract: This study proposes to determine whether the use of interactive computer animations, in the role of previous organizers, may favor the processes of teaching and learning of the concepts of mechanical energy. To that end, we developed an interactive animation, called the Energia no Bate - Estaca, and employed a study that evaluated the influence of the use of this feature with students from a public school high school. The results obtained suggest that the use of interactive animations as previous organizers is feasible and effective to promote the teaching-learning process of the concepts of mechanical energy.

Keywords: animation interactive; simulation; energy; teaching of physics.

\section{Introdução}

Trabalhos como Tavares e Santos (2003), Balen e Netz (2005) e Borcelli e Costa (2008) e Costa et al (2010) e numerosos outros, apontam que o uso de recursos da informática como ferramentas didáticas, pode trazer significativos benefícios aos processos de ensino e aprendizagem em Ciências.

Esta pesquisa, na mesma linha dos trabalhos citados anteriormente, se propôs a verificar se o uso de animações interativas computacionais, na função de organizadores prévios, pode favorecer os processos de ensino e aprendizagem dos conceitos da energia mecânica.

As animações interativas, investigadas aqui quanto a sua eficácia no papel de organizadores prévio, são, segundo Borcelli e Costa (2008), filmes de computação gráfica que permitem ao usuário interagir, alterando determinados parâmetros, durante a simulação um evento específico. Esses autores ainda acrescentam que este recurso seria capaz de permitir ao aluno: "interagir, através da manipulação de variáveis que alteram 
o resultado final da simulação, possibilitando a visualização de situações que dificilmente seriam acessíveis em laboratórios didáticos” (Borcelli e Costa, 2008, p. 2).

Tavares e Santos (2003), definem organizadores prévios como recursos empregado para manipular a estrutura cognitiva dos alunos possibilitando o desenvolvimento dos conceitos subsunçores que facilitarão a aprendizagem significativa dos assuntos a serem ainda apresentados.

O destaque dado ao tema da energia neste trabalho justifica-se pelo fato do mesmo ser notoriamente relevante, além da própria Física, na Química e Biologia, onde está presente na quase totalidade dos processos ali discutidos. A lei da conservação da energia, por exemplo, é apresentada por Feynman (2001), como uma lei que governa todos os fenômenos naturais conhecidos até hoje. Kantor (2010) acrescenta que não é possível imaginar uma única situação do cotidiano onde a energia não esteja presente. Assim, a compreensão dos fundamentos básicos envolvidos no estudo da energia é absolutamente imprescindível para que o aluno tenha o correto entendimento dos princípios que regem os fenômenos naturais, presentes no cotidiano, e dos processos utilizados no mundo produtivo.

\section{A Aprendizagem Significativa}

A aprendizagem significativa é o conceito central da teoria criada por David Paul Ausubel (1918-2008), médico especializado em psiquiatria e Professor da Universidade de Colúmbia, em Nova Iorque.

A aprendizagem significativa ocorre quando as novas idéias se relacionam de forma não arbitrária e substantiva com as idéias já existentes na estrutura. A primeira dessas duas condições indica que o novo conceito deve ser disposto de forma lógica e coerente na estrutura cognitiva do indivíduo. A segunda, que se refere à necessidade dos novos conceitos serem incluídos de forma substantiva, Präss (2008) explica que isto significa que o aluno deve apreender o sentido, o significado daquilo que se ensinou. Borcelli e Costa (2008), explicam que na aprendizagem significativa, "informações mais específicas são ligadas a conceitos mais gerais e inclusivos relacionados a esta informação. Estes conceitos pré-existentes servem como ponto de ancoragem para a nova informação”. (Borcelli e Costa, 2008, p. 3).

Aos conceitos preexistentes na estrutura cognitiva do indivíduo, aos quais são conectados os novos, Ausubel (2003), denomina subsunçores. Rosa (2010), afirma que, quando o indivíduo incorpora novos conceitos sem que estes se liguem a um ou mais subsunçores, então se diz que está havendo aprendizagem mecânica.

Neste tipo de aprendizagem as novas idéias não estabelecem uma relação lógica e coerente com os conceitos já presentes na estrutura cognitiva do indivíduo, mas são apenas armazenadas de forma arbitrária e desconexa. Quando o processo ocorre dessa forma, além declaro prejuízo para a longevidade do que é aprendido, em razão da falta de estabilidade da estrutura, o aprendizado não será substantivo.

Präss (2008), explicando as conseqüências dessa falta de substantividade desse aprendizado, afirma que:

[...] o indivíduo não é capaz de expressar o novo conteúdo com linguagem diferente daquela com que foi primeiramente aprendido. De fato, ele não aprendeu o significado, o sentido do novo material, mas tão-somente decorou a seqüência de palavras que o definia. Por conta disso, ele será incapaz de utilizar este conhecimento em contexto diferente daquele no qual fora primeiramente apresentado a estes conceitos/ idéias. (Präss, 2008, p.29). 
Apesar de Ausubel ter destacado as amplas vantagens da aprendizagem significativa em relação à mecânica, ele afirmava que no processo ensino-aprendizagem de determinados conteúdos havia circunstâncias em que a aprendizagem mecânica era necessária e inevitável.

Sobre o papel dos organizadores prévios, Rosa (2010) explica que os conceitos subsunçores, aos quais os novos conceitos devem se conectar para que tenhamos uma aprendizagem significativa, vão sendo construídos ao longo do desenvolvimento do organismo em constante interação com o meio. No entanto, no âmbito escolar, o professor não pode depender unicamente da formação espontânea desses elementos na estrutura cognitiva de modo a viabilizar o ensino. Uma solução para possibilitar a aprendizagem significativa de conteúdos onde os alunos ainda não reúnem os necessários subsunçores consiste na utilização dos organizadores prévios. Rosa (2010) explica que: "Estas ferramentas são uma tentativa de prover a ponte necessária entre a estrutura cognitiva atual e a estrutura do material instrucional que se está querendo ensinar”. (Rosa, 2010, p.105).

Segundo Tavares e Santos (2003), os organizadores prévios são materiais introdutórios que são apresentados antecipadamente e se caracterizam por apresentar um grau de generalidades e de inclusividade em um nível superior ao material principal que será apresentado em seguida. Esses organizadores, estruturados em diversos formatos como textos escritos, figuras, discussões, filmes ou outros, são empregados para "deliberadamente manipular a estrutura cognitiva dos alunos, a fim de que sejam desenvolvidos conceitos subsunçores, capazes de facilitar a aprendizagem significativa dos assuntos a serem ainda apresentados” (Tavares e Santos, 2003, p.3).

A teoria de Ausubel foi eleita como fundamentação deste trabalho, por apontar o distanciamento entre os conceitos presentes na estrutura cognitiva do aluno em relação ao que precisa aprender, como um dos principais problemas para que ocorra a aprendizagem significativa sobre um dado tema. Para atacar esse problema, segundo Rosa (2010), Ausubel propõe o uso de organizadores prévios que, a exemplo do é feito nos trabalhos Tavares e Santos (2003) e Borcelli e Costa (2008), tem aqui o seu papel desempenhado por uma animação interativa.

\section{A metodologia}

Esta pesquisa foi desenvolvida no ano de 2011 em uma escola da rede pública estadual de ensino de São Luis, capital do Maranhão. Foi considerado como a população, o conjunto composto pelos 1650 alunos, que segundo dados do INEP, foram matriculados em 2011 nessa escola, na $2^{\mathrm{a}}$ e $3^{\mathrm{a}}$ série do Ensino Médio, nos três turnos.

Os instrumentos de coleta de dados empregados nesta investigação foram baseados nos trabalhos de Tavares e Santos (2003), Balen e Netz (2005) e Borcelli e Costa (2008), consistindo de testes escritos que, no caso deste trabalho, abordam os conceitos da Energia Mecânica.

Foi tomada como variável dependente a média dos escores obtidos pelos grupos ao serem submetidos ao teste em cada etapa da pesquisa. Essa média, denotada por $\$$, foi definida como a média aritmética dos escores $X_{1}, X_{2}, X_{3} \ldots, X_{N}$, coletados na

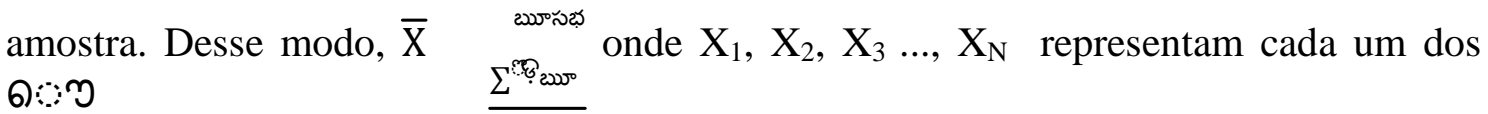

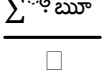

escores coletados. O número de valores de $\mathrm{X}$ é igual ao valor de $\mathrm{N}$, que representa o tamanho da amostra. Um escore $\mathrm{X}$ foi definido como um valor associado ao total de acertos de cada integrante do grupo pesquisado, ao se submeter ao teste. O emprego da 
animação interativa foi tomado como a variável experimental ou variável independente a ser manipulada.

Para verificar a influência da manipulação da variável independente sobre as médias dos escores dos grupos, foi usado o método da diferença ou plano clássico da prova das hipóteses o qual, de acordo com Lakatos e Marconi (2008), estabelece que:

\begin{abstract}
Se em um caso, no qual aparece o fenômeno que se investiga e em outro caso, no qual não aparecem, as circunstâncias são todas comuns, exceto uma, apresentando-se essa no primeiro, a circunstância única pela quais ambos os casos diferem é o efeito, ou a causa, ou uma parte indispensável do dito fenômeno. (Lakatos e Marconi, 2008, p. 233).
\end{abstract}

Para viabilizar esse plano de prova das hipóteses, as autoras acima destacam que este método exige dois grupos semelhantes entre si. Um deles é denominado experimental e é nele que manipularemos a variável independente. O outro, denominado grupo controle, passará por todas as etapas a que foi submetido o grupo experimental, excetuando-se apenas aquela onde a variável independente será manipulada. A importância do grupo controle para esse método fica evidenciada quando Campbell e Stanley (1979) alertam que eventos específicos, além da manipulação da variável experimental, podem ocorrer entre a primeira e a segunda medida. Essa variável estranha, denominada pelos autores de história, pode comprometer a validade do estudo já que seus efeitos podem ser confundidos com os efeitos da variável experimental. Porém, segundo Campbell e Stanley (1979), a variável história é controlada pelo método na medida em que eventos particulares, que possam produzir uma variação na média dos escores do grupo experimental, produziriam isto também no grupo controle. Para assegurar que o grupo experimental e o de controle estejam expostos à mesma história, segundo esses autores, no momento da aplicação dos testes, os integrantes de cada grupo não devem estar separados segundo o grupo ao qual pertençam, mas reunidos de forma aleatória.

Assim, conforme Lakatos e Marconi (2008), supõe-se que a diferença entre os escores antes e depois, no grupo de controle, seja o resultado da ação das variáveis estranhas. No grupo experimental, a diferença entre os escores antes e depois, corresponde à ação da variável experimental adicionada aos efeitos das mesmas variáveis estranhas que atuaram sobre o grupo de controle.

A partir da escolha ao acaso dos alunos na população, foram compostos os dois grupos da pesquisa; o experimental (grupo E), com 52 alunos e o de controle (grupo C), com 54.

Como parte da metodologia empregada nesta investigação, optamos por desenvolver as atividades em quatro etapas, sendo que a primeira, a etapa inicial, teve a função de encontrar os escores dos grupos E e C em seu estado inicial.

Nesta primeira etapa e na última, a exemplo do ocorre em Balen e Netz (2005), as questões foram corrigidas segundo o seguinte critério:

a) CT - Compreensão total. As respostas foram assim classificadas quando o aluno respondeu de acordo com o modelo aceito pelo formalismo da Física;

b) CP - Compreensão Parcial, quando a resposta foi parcialmente correta, apresentando falhas nos conceitos ou no tratamento matemático empregado;

c) CE - Concepção Errônea, quando o aluno respondeu utilizando argumentos inadequados ou quando o estudante não respondeu.

Com a finalidade de quantificar os resultados, definimos o escore $\mathrm{X}$ de cada participante como o resultado da expressão $X=1 \mathrm{~N}_{\mathrm{CT}}+0,5 \mathrm{~N}_{\mathrm{CP}}$, onde $\mathrm{N}_{\mathrm{CT}}$ e $\mathrm{N}_{\mathrm{CP}}$ representam os números de respostas classificadas como $\mathrm{CT}$ e $\mathrm{CP}$, respectivamente. Cabe destacar que CT vale 1,0, CP vale 0,5 e CE vale 0 , valores definidos como a 
pontuação conferida para a parcela de respostas correta, ou seja, CT equivale a $100 \%$ correto, de modo que essa parcela corresponde a 1 , enquanto $\mathrm{CP}$ equivale a $50 \%$ correto, significando que essa parcela corresponde a 0,5.

Para aplicação do teste, os alunos foram separados em três salas de aulas, independente do grupo ao qual pertenciam, e durante duas aulas de 50 minutos responderam às questões.

A segunda etapa desta pesquisa, aplicada somente ao grupo experimental (E), consistiu no emprego de animações interativas como organizadores prévios, para introduzir conceitos sobre a Energia Mecânica. Nesta etapa, o grupo E primeiramente assistiu, no auditório da escola, a um pequeno vídeo caseiro ${ }^{1}$, com duração de 114 segundos, que mostra uma descida realizada em uma grande montanha russa. $\mathrm{O}$ propósito desse vídeo é despertar o interesse do aluno para o conteúdo que seria tratado posteriormente e com isso criar um ambiente onde ele se sinta mais motivado a se envolver com a atividade propostas, facilitando a conexão dos novos conceitos à sua estrutura cognitiva. A motivação do aluno para conectar os novos conceitos aos subsunçores é considerada por Rosa (2010) como um elemento indispensável para que ocorra a aprendizagem significativa com base na teoria de Ausubel, de modo que a apresentação de vídeos, como o indicado aqui, assumiu uma destacada relevância para o desenvolvimento deste trabalho. Em seguida, ainda no mesmo espaço físico, foi apresentada ao grupo a animação interativa utilizada nesta pesquisa: a Energia no Bateestaca (figura 1), uma animação que desenvolvemos exclusivamente para este trabalho.

Essa animação, que aborda conceitos sobre Energia Mecânica, foi desenvolvida em linguagem Visual Basic (VB), no ambiente Visual Basic 6 da Microsoft. Nela podemos simular o processo de transformação de energia que ocorre em um bate-estaca, equipamento que em função das várias construções em andamento em São Luís são vistos pelos alunos em diversos pontos da cidade, constituindo, portanto, um elemento de seu mundo vivencial.

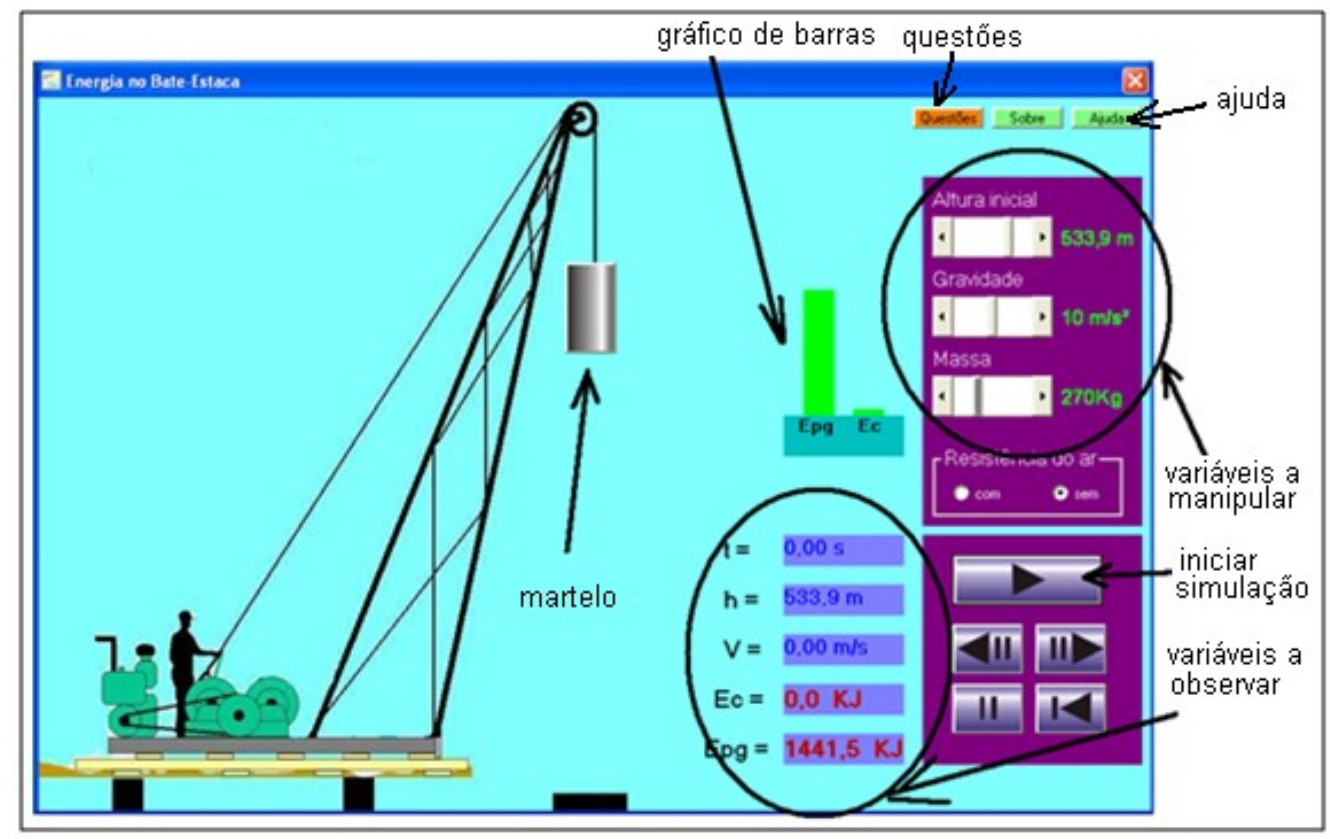

Figura 1 - Tela inicial da animação interativa Energia no Bate-Estaca.

\footnotetext{
${ }^{1}$ Esse vídeo sobre a montanha russa pode ser encontrado no endereço http://www.youtube.com/watch?v=DFZ_hve5K2s. Acesso em 25/04/2011.
} 
Para as simulações, determinamos a altura da qual o martelo será abandonado, a aceleração da gravidade local, a massa do martelo e podemos decidir também se queremos considerar ou não a resistência do ar. Quando é iniciada a simulação, o aluno pode acompanhar a evolução temporal da altura e velocidade do martelo, assim como o valor da energia cinética e da energia potencial gravitacional disponível no sistema.

A opção pelo desenvolvimento de nossa própria animação decorreu do fato das opções disponíveis nos repositórios não apresentarem um grau de interatividade adequado para as condições que o trabalho demandava.

A exibição do vídeo da montanha russa e a apresentação da animação interativa ao grupo experimental ocorreram no mesmo dia e no mesmo local, utilizando para tanto, dois horários de 50 minutos.

Nos dois dias seguintes, utilizando os dois laboratórios de informática da escola durante dois horários de 50 minutos, os alunos, organizados em duplas, foram orientados a explorar a animação. Foi recomendado que iniciassem a exploração pela função Ajuda do programa, que traz um roteiro mostrando como ajustar os parâmetros para produzir as simulações.

Em seguida, o grupo foi estimulado a ler as questões que a animação propõe e resolve-las através de simulações realizadas no próprio programa. O formulário de questões da animação, acessado através de um dos botões do canto superior direito, contêm as seguintes perguntas:

1. Que tipo de energia o bloco ganha quando é levantado?

2. Essa energia depende do valor de quais grandezas?

3. Quando o bloco estiver parado a certa altura ele possuirá energia cinética (Ec)? Por quê?

4. Quando o bloco estiver parado a certa altura e aumentarmos sua massa, ocorrerá modificação em qual das energias?

5. Quando o bloco começar a cair, qual energia irá diminuir à medida que o bloco cai? Por que diminui?

6. À medida que o bloco cair, qual energia irá aumentar? Por que aumenta?

7. Se não considerarmos a resistência do ar, a soma das duas energias será sempre a mesma à medida que o corpo cai?

8. Se considerarmos a resistência do ar, a soma das duas energias será sempre a mesma à medida que o corpo cai?

Durante a apresentação e a exploração da animação não foi fornecida qualquer definição ou equação matemática sobre os conceitos ali envolvidos. Foi esclarecido que o aluno deveria descobrir as relações entre esses conceitos através de simulações feitas na própria animação. Foi recomendado que para responder as questões 1, 2 e 4, o aluno precisava elevar o martelo do bate-estaca, manipulando a variável altura inicial, e, deixando-o parado a certa altura, observar o gráfico de barras. Com o martelo ainda parado a certa altura, o aluno deveria modificar as variáveis gravidade e massa do martelo e observar as consequências disso no gráfico de barras e extrair as suas conclusões.

Nas questões 3, 5 e 6, estando ainda o martelo parado a certa altura, o aluno deveria acionar o botão de início da simulação e observar no gráfico de barras e no campo das variáveis, o comportamento dos valores da altura, velocidade, energia cinética e energia potencial gravitacional. O aluno deveria observar qual energia cresce e qual decresce à medida que o corpo cai, e relacionando com a simulação anterior, concluir do que depende cada uma delas.

Para a questão 7, o aluno deveria colocar o martelo a certa altura e mantendo-o 
ali parado, anotar os valores da energia cinética e energia potencial gravitacional naquele ponto. Em seguida, acionar o início simulação, permitindo que o martelo caísse, e pausar o movimento em vários pontos, anotando os valores das duas energias em cada um deles. Para cada ponto, o aluno deveria somar as duas energias, encontrando a energia total em cada um deles e comparar os resultados. Inicialmente essa simulação deve ser realizada desconsiderando a resistência do ar, marcando esta opção na animação. Para a questão 8, o mesmo procedimento acima deveria ser realizado, porém, levando agora em consideração a resistência do ar.

$\mathrm{Na}$ terceira etapa, os alunos dos grupos $\mathrm{E}$ e C novamente foram separados em três salas, T1, T2 e T3, sendo que as duas primeiras foram compostas por 18 alunos do grupo E e 17 do grupo C, T3 foi formada por 18 alunos de cada grupo. Durante duas aulas de 50 minutos, em cada um dos dois dias destinados para essa etapa, os alunos receberam aulas expositivas sobre os conceitos da Energia Mecânica. As aulas foram ministradas nesses dois encontros por três professores de Física, usando como recurso quadro branco e projetor multimídia. No primeiro dia foi abordado o tema Energia e Trabalho (definições fundamentais, energia cinética e energia potencial gravitacional). No segundo dia, Conservação da energia (a energia mecânica e o princípio da conservação da energia mecânica).

Na última etapa, os dois grupos E e C foram novamente submetidos ao mesmo teste da etapa inicial e corrigidos segundo os critérios já apresentados.

O resumo do cronograma de execução das etapas é mostrado no quadro 1.

Quadro 1 - Etapas de desenvolvimento da pesquisa.

\begin{tabular}{|c|c|c|c|c|}
\hline ETAPA & DESCRIÇÃO & DIA & GRUPO & DURAÇÃO \\
\hline $\begin{array}{l}1^{\mathrm{a}}-\mathrm{ETAPA} \\
\text { INICIAL }\end{array}$ & $\begin{array}{l}\text { Aplicação do } \\
\text { teste inicial }\end{array}$ & $1^{\circ}$ & E e C & $2 \times 50 \mathrm{~min}$ \\
\hline \multirow{3}{*}{$\begin{array}{l}2^{\mathrm{a}}-\text { USO DA } \\
\text { ANIMAÇÃO } \\
\text { INTERATIVA }\end{array}$} & $\begin{array}{l}\text { Vídeo montanha } \\
\text { russa }\end{array}$ & $2^{\circ}$ & $\mathrm{E}$ & $2 \times 50 \min$ \\
\hline & \multirow{2}{*}{$\begin{array}{c}\text { Uso da } \\
\text { animação } \\
\text { interativa }\end{array}$} & $3^{\circ}$ & $E$ & $2 \times 50 \mathrm{~min}$ \\
\hline & & $4^{\circ}$ & $E$ & $2 \times 50 \mathrm{~min}$ \\
\hline \multirow{2}{*}{$\begin{array}{l}3^{a}-\text { AULA } \\
\text { EXPOSITIVA }\end{array}$} & \multirow{2}{*}{$\begin{array}{c}\text { Aulas sobre os } \\
\text { conceitos da } \\
\text { energia } \\
\text { mecânica }\end{array}$} & $5^{\circ}$ & E e C & $2 \times 50 \mathrm{~min}$ \\
\hline & & $6^{\circ}$ & E e C & $2 \times 50 \mathrm{~min}$ \\
\hline $\begin{array}{l}4^{\mathrm{a}}-\mathrm{ETAPA} \text { DE } \\
\text { VERIFICAÇÃ̃O }\end{array}$ & $\begin{array}{l}\text { Aplicação do } \\
\text { teste de } \\
\text { verificação }\end{array}$ & $7^{\circ}$ & E e C & $2 \times 50 \mathrm{~min}$ \\
\hline
\end{tabular}

Para a formulação e julgamento das hipóteses da pesquisa, foram definidos os parâmetros $\mathrm{X}_{\mathrm{Ei}}$ e $\mathrm{X}_{\mathrm{Ef}}$ como as medidas inicial e final dos escores do grupo experimental e $\mathrm{X}_{\mathrm{Ci}}$ e $\mathrm{X}_{\mathrm{Cf}}$ como as medidas inicial e final dos escores do grupo controle. Definiu-se

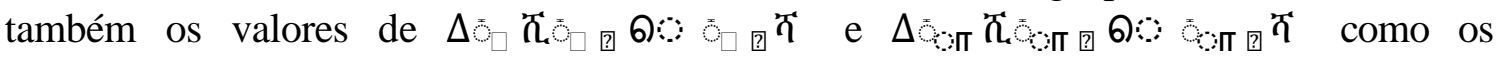
escores diferenciais de cada elemento dos grupos controle e experimental, respectivamente. Para a análise dos escores diferencias de cada grupo foi realizado um teste $\mathrm{t}$ de Student para as médias de duas amostras independentes. Este teste é indicado, segundo Guimarães (2008), quando desejamos comparar as médias de duas amostras distintas. Assim, foram definidas as hipóteses:

$$
\begin{gathered}
\mathrm{H}_{0}: \Delta \mathrm{X}_{\mathrm{E}} \text { ๑๐ } \\
\Delta \mathrm{X}_{\mathrm{C}} \\
\mathrm{H}_{1}: \Delta \mathrm{X}_{\mathrm{E}} \text { 田 } \Delta \mathrm{X}_{\mathrm{C}}
\end{gathered}
$$


A hipótese nula $\mathrm{H}_{0}$ assume que os escores diferenciais dos grupos experimental e de controle são iguais. A aceitação dessa hipótese negaria a ação da variável independente, a animação interativa, sobre a média dos escores do grupo experimental. A hipótese alternativa $\mathrm{H}_{1}$ contesta a hipótese inicial e assume que o escore diferencial do grupo experimental é maior que o do grupo controle. Para o julgamento das hipóteses, foi calculado o parâmetro t de Student ( $\mathrm{H}_{\square}$ ( $)$ que foi calculado através da expressão:

$$
=\frac{\overline{\Delta X} \bar{\Delta}}{\omega \frac{s_{\square}^{\text {6ी }}}{N_{\square}}}
$$

$N_{E}$ e $N_{C}$ representam os números de medidas de $\Delta \_$em cada um dos dois grupos. Os parâmetros $\varsigma_{\text {m }}$ e $\boldsymbol{s}_{\square}$ são os desvios padrão dos escores diferenciais dos grupos experimental e controle, respectivamente.

Conforme Guimarães (2008), além de calcular a estatística $\mathbf{N}_{\square \square}$, devemos escolher o nível de significância ? do teste - parâmetro que Spiegel (1985) define como a probabilidade de se cometer o erro de rejeitar a hipótese inicial $\mathrm{H}_{0}$ quando ela é verdadeira. Spiegel (1985) ainda esclarece que, embora ? seja uma escolha do pesquisador, normalmente são utilizados os valores $1 \%, 5 \%$ ou $10 \%$. Por ser o valor mais usado, optou-se neste trabalho por empregar um nível de significância de $5 \%$ para os testes de forma que tenhamos uma confiança de $95 \%$ de que a decisão sobre $\mathrm{H}_{0}$ não será equivocada. Guimarães (2008), explica que o julgamento das hipóteses é feito comparando-se o valor de $t_{c a l}$ com o valor crítico do teste, representado por $m_{\forall}$. O valor crítico do teste $=_{ఈ}$ representa o limite da região de aceitação de H0, como pode ser visto na figura a seguir.

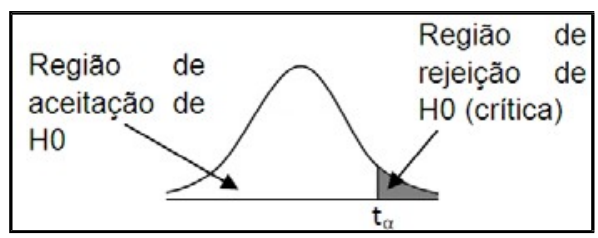

Figura 2 - Região de rejeição de $\mathrm{H0}$ em teste unilateral Fonte: Guimarães, 2008, p. 96 (adaptado).

Em outras palavras, para o teste em questão, se $t_{c a l}>w_{\varpi_{0}}$, dizemos que a variável do teste caiu na região crítica e dessa forma há evidências estatísticas para rejeitar $\mathrm{H}_{0}$; caso contrário, $\mathrm{H}_{0}$ será aceita. $\uplus_{\uplus_{3}}$ é encontrado na tabela de distribuição de t, considerando o nível de significância ? e o número de graus de liberdade GL.

\section{Resultados}

Na tabelas 1 são mostrados os valores encontrados para a média dos escores, desvio padrão e variância para cada um dos dois grupos. Na Tabela 1 esses parâmetros foram obtidos na etapa inicial da pesquisa, antes do emprego da animação interativa. 
Tabela 1 - Grupos E e C, Etapa inicial, médias e dispersão.

\begin{tabular}{|c|c|c|c|c|c|c|c|}
\hline Grupo & Etapa & $\begin{array}{c}\text { Tamanho } \\
(\mathrm{N})\end{array}$ & $\begin{array}{c}\text { Escore } \\
\text { Min }\end{array}$ & $\begin{array}{c}\text { Escore } \\
\text { Max }\end{array}$ & $\begin{array}{c}\text { Méd } \\
\overline{\mathrm{R}}\end{array}$ & $\begin{array}{c}\text { D.Pad. } \\
(\mathrm{s})\end{array}$ & $\begin{array}{c}\text { Variância } \\
\left(\mathrm{s}^{2}\right)\end{array}$ \\
\hline E & INICIAL & 52 & 0 & 11 & 2,88 & 2,44 & 5,96 \\
\hline C & INICIAL & 54 & 0 & 9,5 & 2,37 & 1,84 & 3,38 \\
\hline E & Verificação & 52 & 2,5 & 12 & 4,70 & 2,50 & 6,25 \\
\hline C & Verificação & 54 & 1 & 13 & 3,62 & 2,30 & 5,30 \\
\hline
\end{tabular}

A estatística ou variável do teste $t_{c a l}$ foi encontrada através do procedimento de cálculo já descrito, obtendo-se $t_{c a l}=\mathbf{1 , 9 2 5}$. O valor crítico ( ${ }_{\uplus}$ ), encontrado na tabela de distribuição de $t$, com base no nível de significância adotado e no número de graus de liberdade ( $\mathrm{GL}=\mathrm{N}_{\mathrm{A}}-1$ ), foi 1,645. Como o valor $t_{\text {cal }}$ encontra-se além do limite ${ }^{-}$, verificamos que a estatística do teste caiu na região de rejeição de $H_{0}$. Assim, concluímos que há evidências estatísticas para assumir que houve um ganho em escores maior no grupo experimental em relação ao grupo controle, provavelmente em decorrência da utilização das animações interativas.

\section{Conclusões}

Verificou-se nesse teste que o grupo experimental apresentou um ganho em escores superior ao grupo de controle, sendo que o processo ao qual foram submetidos diferiu pelo fato de somente o grupo experimental ter passado pela etapa de utilização das animações interativas. Assim, é possível concluir por meio do resultado do teste estatístico empregado, a um nível de significância de $5 \%$, que a superioridade dos escores diferencias do grupo experimental foi consequência, provavelmente, do uso das animações interativas.

Evidentemente, outras variáveis como as diferenças de motivação dos grupos, que não são consideradas ou isoladas nesta metodologia, podem trazer implicações importantes aos resultados da pesquisa. Ainda assim, em face dos resultados observados neste trabalho e que corroboram os obtidos por outros pesquisadores em investigações semelhantes mencionadas aqui, consideramos plausível concluir que as animações interativas, na função de organizadores prévios, podem favorecer os processos de ensino e de aprendizagem dos conceitos da Energia Mecânica que foram explorados ao longo deste trabalho.

Ao possibilitarmos que os estudantes se envolvessem com ferramentas computacionais interativas e pudessem manipular e testar os parâmetros físicos envolvidos acreditamos estar proporcionando meios para que pudessem construir novos conhecimentos e, com isso, ampliar a sua capacidade de atuação autônoma diante das situações exploradas.

Considerando a importância do tema abordado para o ensino de Ciências, julgamos que merece ser considerada a possibilidade de desenvolvimento de trabalhos semelhantes empregando outros recursos computacionais com uma orientação teórica diferente, como tutoriais ou jogos, para que se possa ampliar a base de dados e os conhecimentos obtidos, fortalecendo a convicção de que estes recursos computacionais podem efetivamente contribuir para a aprendizagem dos estudantes. 


\section{Referências bibliográficas}

AUSUBEL, D. P. Aquisição e retenção de conhecimento: uma perspectiva cognitiva. Lisboa: Plátano, 2003.

AUSUBEL, D. P.; NOVAK, J. D.; HANESIAN, J. Psicologia Educacional. Rio de Janeiro: Interamericana, 1980.

BALEN, O.; NETZ, P. A. Utilizando a modelagem e a simulação computacional no estudo do comportamento dos gases. In: XVI Simpósio Nacional de Ensino de Física, p. 1-4, 2005.

BORCELLI, A. F.; COSTA, S. S. C. D. Animação Interativa: um material potencialmente significativo para a aprendizagem de conceitos em física. XI Encontro de Pesquisa em Ensino de Física. Curitiba: [s.n.]. 2008.

BRASIL. Ministério da Educação; INEP - Dataescolabrasil. Sistema de estatísticas educacionais. Disponível em: <http://www.dataescolabrasil.inep.gov.br>. Acesso em 29 abr. 2011.

CAMPBELL, D. T.; STANLEY, J. C. Delineamentos experimentais e quaseexperimentais de pesquisa. São Paulo: EPU/Edusp, 1979.

COSTA, H. R. et al. Desenvolvimento e aplicação de um software educacional na aprendizagem significativa do estudo dos gases. $50^{\circ}$ Congresso Brasileiro de Química. Cuiabá: CBQ. 2010.

FEYNMAN, Richard. Física em seis lições. 2.ed. Rio de Janeiro: Ediouro, 1999. GUIMARÃES, P. R. B. Métodos Quantitativos Estatísticos. IESDE Brasil S.A., Curitiba, 2008.

KANTOR, C. A. et. al. Física $\mathbf{1}^{\mathbf{0}}$ Ano (coleção Quanta Física; v.1). 1ª Edição. São Paulo: Editora PD, 2010.

LAKATOS, Eva Maria; MARCONI, Marina de Andrade. Metodologia Científica. 5. ed. São Paulo: Atlas, 2008.

MORCILLO, A. M. Teste t Student. Campinas: Universidade Estadual de Campinas Faculdade de Ciências Médicas, 2004.

PRÄSS, A. R. Teorias da aprendizagem. Porto Alegre. 2008.

ROSA, P. R. D. S. Instrumentação para o ensino de ciências. Campo Grande: Editora UFMS, 2010.

SPIEGEL, M. R. Estatística. 2. ed. São Paulo: Mcgraw-Hill, 1985.

TAVARES, R.; SANTOS, J. N. D. Organizador prévio e animação interativa. IV International meeting on meaningful learning. Maragogi: [s.n.]. 2003. 